\title{
AN IMPROVED METHOD FOR NONSTATIONARY SIGNALS COMPONENTS EXTRACTION BASED ON THE ICI RULE
}

\author{
Jonatan Lerga, Victor Sucic* \\ Faculty of Engineering, University of Rijeka \\ Vukovarska 58, HR-51000 Rijeka, Croatia \\ jlerga@riteh.hr; vsucic@riteh.hr
}

\author{
Boualem Boashash \\ College of Engineering, Qatar University \\ PO Box: 2713, Doha, Qatar \\ boualem@qu.edu.qa
}

\begin{abstract}
This paper proposes an improved adaptive algorithm for components localization and extraction from a noisy multicomponent signal time-frequency distribution (TFD). The algorithm, based on the intersection of confidence intervals (ICI) rule, does not require any a priori knowledge of signal components and their mixture. Its efficiency is significantly enhanced by using high resolution and reduced cross-terms TFDs. The obtained results are compared for different signal-to-noise ratios (SNRs) and various time and lag window types used in the modified B-distribution (MBD) calculation, proving the method to be a valuable tool in noisy multicomponent signals components extraction in the time-frequency (TF) domain.
\end{abstract}

\section{INTRODUCTION}

Real-life signals, such as those found in telecommunications, radar, sonar, biomedical measurements or seismology, are often nonstationary, meaning that their frequency contest exhibits time-varying properties. In practice, those signals are often also corrupted by additive noise so that signal processing algorithms performances normally depend on the signal-to-noise ratio (SNR).

Since the late 1980's, the time-frequency (TF) signal analysis has been intensively studied for the purpose of finding adequate tools for analyzing non-stationary signals. A special attention was dedicated to multicomponent signals TFDs due to the interactions among signal components (cross-terms) which carry redundant information and may obscure signal features of interest.

An important TFD property is its TF resolution. Good time and frequency resolution seem to be conflicting requirements, hence research efforts are still being deployed to modify existing TFDs or design new TFDs with reduced cross-terms while keeping high TF resolution [1]. Consequently, various reduced interference distributions (RIDs) have been proposed, one of the recent ones being the modified B-distribution (MBD) [2].

*This work is a part of the research project "Optimization and Design of Time-Frequency Distributions", No. 069-0362214-1575, which is financially supported by the Ministry of Science, Education and Sports of the Republic of Croatia.
Furthermore, when dealing with multicomponent signals, the components extraction often precedes other signal processing procedures, such as for example the components instantaneous frequency (IF) estimation [3]. Numerous components extraction procedures have been proposed, often termed as blind source separation (BSS) techniques [4].

Unlike in [5] where the fixed size bandwidths based BSS algorithm was used, in the here proposed method we have introduced adaptivity in the bandwidth size selection. This has resulted in improved components localization and extraction from a noisy multicomponent signal TFD.

\section{COMPONENTS EXTRACTION PROCEDURE}

In this section, we present an improved components selection and extraction algorithm from a noisy multicomponent signal based on the adaptive bandwidth size calculated for each time instant of the signal TFD.

\subsection{The algorithm}

The algorithm (see the flowchart in Fig. 1) has several major steps:

- Step 1: TFD $\rho(t, f)$ calculation

- Step 2: Finding the $\rho(t, f)$ maximum location $\left(t_{0}, f_{0}\right)$ and the TFD slice $\rho\left(t_{0}, f\right)$ selection

- Step 3: Adaptive bandwidth detection (for each time instant $t_{0}$ and each component apart)

- Step 4: The selected bandwidth (defined by its boundaries $\left.\left[f_{0}\left(t_{0}\right)-f_{l}\left(t_{0}\right), f_{0}\left(t_{0}\right)+f_{r}\left(t_{0}\right)\right]\right)$ is used to localize the considered component for the time instant $t_{0}$, (where $f_{0}\left(t_{0}\right)$ is the frequency for which the TFD slice $\rho\left(t_{0}-1, f\right)$ reaches its maximum). Note that $\rho\left(t_{0}-1, f\right)$ is used in order to insure the IF continuity, $f_{l}\left(t_{0}\right)$ and $f_{r}\left(t_{0}\right)$ are the adaptive varying bandwidth lengths calculated using the intersection of confidence (ICI) rule for each time instant apart [3].

- Step 5: Once the component is localized for the time instant $t_{0}$, it is extracted from the TFD so that $\rho\left(t_{0}, f\right)$ is set to zero for $f \in\left[f_{0}\left(t_{0}\right)-f_{l}\left(t_{0}\right), f_{0}\left(t_{o}\right)+f_{r}\left(t_{0}\right)\right]$. 
The above procedure is then repeated for each time instant of $\rho(t, f)$ as long as the $\rho\left(t_{0}, f\right)$ maximum in $f_{0}\left(t_{0}\right)$ vicinity $\left[f_{0}\left(t_{0}\right)-f_{l}\left(t_{0}\right), f_{0}\left(t_{o}\right)+f_{r}\left(t_{0}\right)\right]$ is larger than the preset threshold value $\epsilon_{c}$ (chosen so that only a component is extracted, while avoiding selecting noise as the component) defined as a percentage of the TFD maximum. This stage of the algorithm results in one extracted component. In order to extract the remaining components, the algorithm steps 2-5 are recursively repeated, providing the remaining TFD energy is larger than the preset threshold $\epsilon_{d}$ (defined as a percentage of the total TFD energy).

\subsection{TFD choice}

Due to the presence of a undesirable interference terms, the choice of an appropriate TFD for a given multicomponent signal representation in the $(t, f)$ domain is of crucial importance for the components extraction efficiency. Furthermore, the TFD is expected to have high TF resolution. In general, there exists a tradeoff between those two TFD features [2] which led to various RIDs, one being the MBD, which was shown to outperform other fixed-kernel TFDs in terms of cross-terms reduction and resolution enhancement [2]:

$$
\begin{array}{r}
M B D_{z}(t, f)=\int_{-\infty}^{+\infty} \int_{-\infty}^{+\infty} \frac{\cosh ^{-2 \beta}(t-u)}{\int_{-\infty}^{+\infty} \cosh ^{-2 \beta} \xi d \xi} . \\
\cdot z\left(u+\frac{\tau}{2}\right) \cdot z^{*}\left(u-\frac{\tau}{2}\right) \cdot e^{-j 2 \pi f \tau} d u d \tau
\end{array}
$$

where the parameter $\beta$ controls the TF resolution and cross term suppression $(0<\beta \leq 1)$.

Although the MBD was used in this paper due to its prominent properties in the multicomponent signal TF representation, other high TF resolution and reduced cross-terms TFDs can be also used with the signal components extraction algorithm proposed in this paper.

\subsection{Adaptive bandwidth size selection method}

For the adaptive bandwidth size calculation we have used a method based on the ICI rule [6]. The method's first step (see the flowchart in Fig. 2) is to find $f_{0}$ for each TFD slice $\rho\left(t_{0}, f\right)$ such that the slice $\rho\left(t_{0}-1, f\right)$ reaches its maximum in $\left(t_{0}-1, f_{0}\right)$. Once $f_{0}$ is found, the method calculates the subbandwidths $f_{l}^{+}\left(t_{0}\right)$ and $f_{r}^{+}\left(t_{0}\right)$ which form the overall bandwidth for the considered time instant $t_{0}$, defined as $\left[f_{0}\left(t_{0}\right)-f_{l}^{+}\left(t_{0}\right), f_{0}\left(t_{0}\right)+f_{r}^{+}\left(t_{0}\right)\right]$.

In order to get the subbandwidths $f_{l}\left(t_{0}\right)$ and $f_{r}\left(t_{0}\right)$, the ICI rule introduces two sequences of growing subbandwidths lengths to the left hand and the right hand side of $f_{0}$, denoted as $K_{l}$ and $K_{r}$, where $H_{l}\left(t_{0}, k_{l}\right)=\left\{f_{l}\left(t_{0}, 1\right)<f_{l}\left(t_{0}, 2\right)<\right.$ $\left.\cdots<f_{l}\left(t_{0}, K_{l}\right)\right\}$ and $H_{r}\left(t_{0}, k_{r}\right)=\left\{f_{r}\left(t_{0}, 1\right)<f_{r}\left(t_{0}, 2\right)<\right.$ $\left.\cdots<f_{r}\left(t_{0}, K_{r}\right)\right\}$. The accompanying confidence intervals are:

$$
\begin{array}{cc}
D_{l}\left(t_{0}, k_{l}\right)=\left[L_{l}\left(t_{0}, k_{l}\right), U_{l}\left(t_{0}, k_{l}\right)\right], & 1 \leq k_{l} \leq K_{l}, \\
D_{r}\left(t_{0}, k_{r}\right)=\left[L_{r}\left(t_{0}, k_{r}\right), U_{r}\left(t_{0}, k_{r}\right)\right], & 1 \leq k_{r} \leq K_{r},
\end{array}
$$

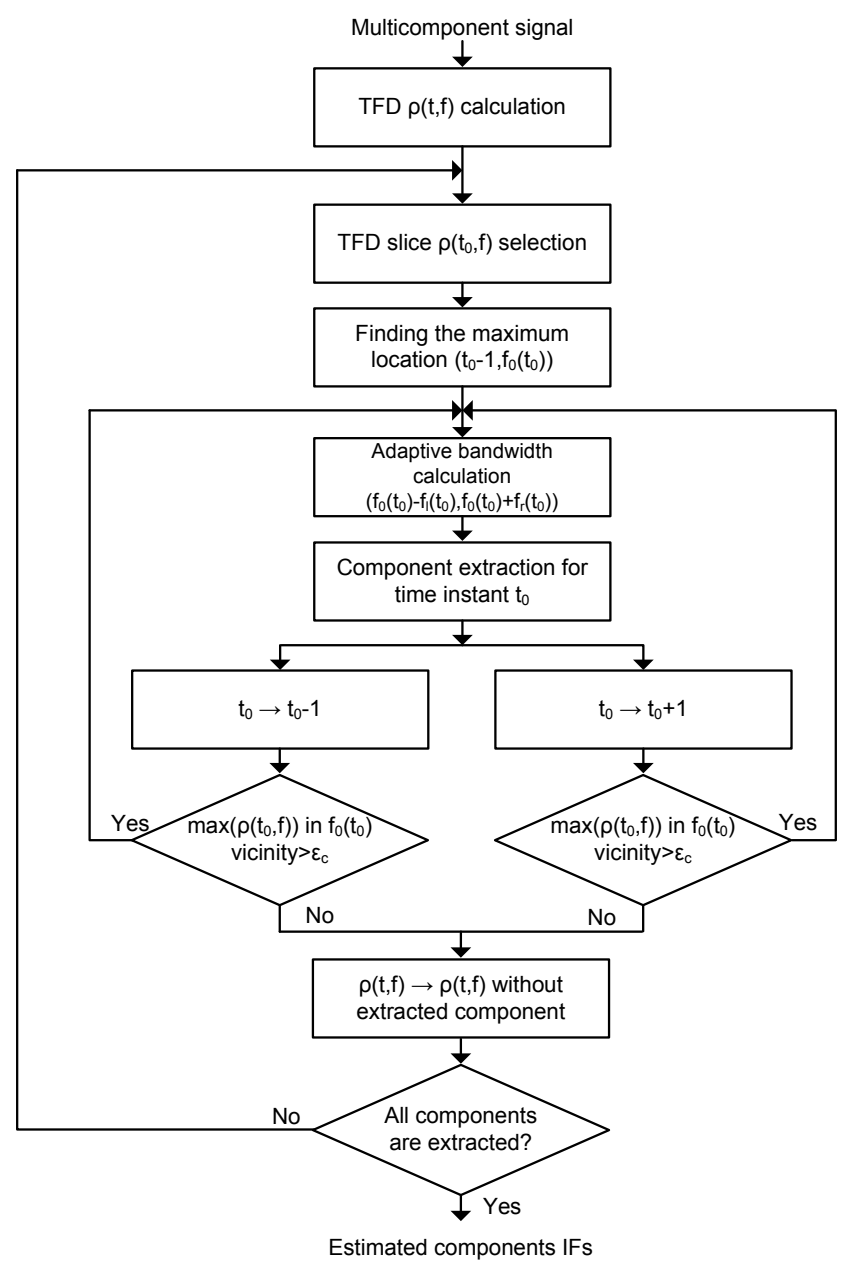

Fig. 1. Components extraction flowchart.

where the lower limits $L_{l}\left(t_{0}, k_{l}\right)$ and $L_{r}\left(t_{0}, k_{r}\right)$ and the upper limits $U_{l}\left(t_{0}, k_{l}\right)$ and $U_{r}\left(t_{0}, k_{r}\right)$ (the indices $l$ and $r$ stand for the left hand and the right hand side of $f_{0}$ ), are respectively defined as:

$$
\begin{aligned}
L_{l}\left(t_{0}, k_{l}\right) & =\rho\left(t_{0}, f_{0}\right)-\Gamma \sigma\left(t_{0}, k_{l}\right), \\
L_{r}\left(t_{0}, k_{r}\right) & =\rho\left(t_{0}, f_{0}\right)-\Gamma \sigma\left(t_{0}, k_{r}\right), \\
U_{l}\left(t_{0}, k_{l}\right) & =\rho\left(t_{0}, f_{0}\right)+\Gamma \sigma\left(t_{0}, k_{l}\right), \\
U_{r}\left(t_{0}, k_{r}\right) & =\rho\left(t_{0}, f_{0}\right)+\Gamma \sigma\left(t_{0}, k_{r}\right) .
\end{aligned}
$$

The parameter $\Gamma$ regulates the confidence interval width (where the probability $P(\Gamma) \rightarrow 1$ as $\Gamma$ grows), and $\sigma\left(t_{0}, k\right)$ is the standard deviation of the estimated $\hat{\rho}\left(t_{0}, f_{0}\right)$ value [6]. The ICI rule then tracks the values of the largest lower and the smallest upper confidence intervals limits for each sequence of confidence intervals $D_{l}\left(t_{0}, k_{l}\right)$ and $D_{r}\left(t_{0}, k_{r}\right)$, defined as:

$$
\begin{aligned}
\bar{L}_{l}\left(t_{0}, k_{l}\right) & =\max _{i=1, \ldots, k_{l}} L_{i}\left(t_{0}, k_{l}\right), \\
\underline{U}_{l}\left(t_{0}, k_{l}\right) & =\min _{i=1, \ldots, k_{l}} U_{i}\left(t_{0}, k_{l}\right), \\
\bar{L}_{r}\left(t_{0}, k_{r}\right) & =\max _{i=1, \ldots, k_{r}} L_{i}\left(t_{0}, k_{r}\right), \\
\underline{U}_{r}\left(t_{0}, k_{r}\right) & =\min _{i=1, \ldots, k_{r}} U_{i}\left(t_{0}, k_{r}\right),
\end{aligned}
$$




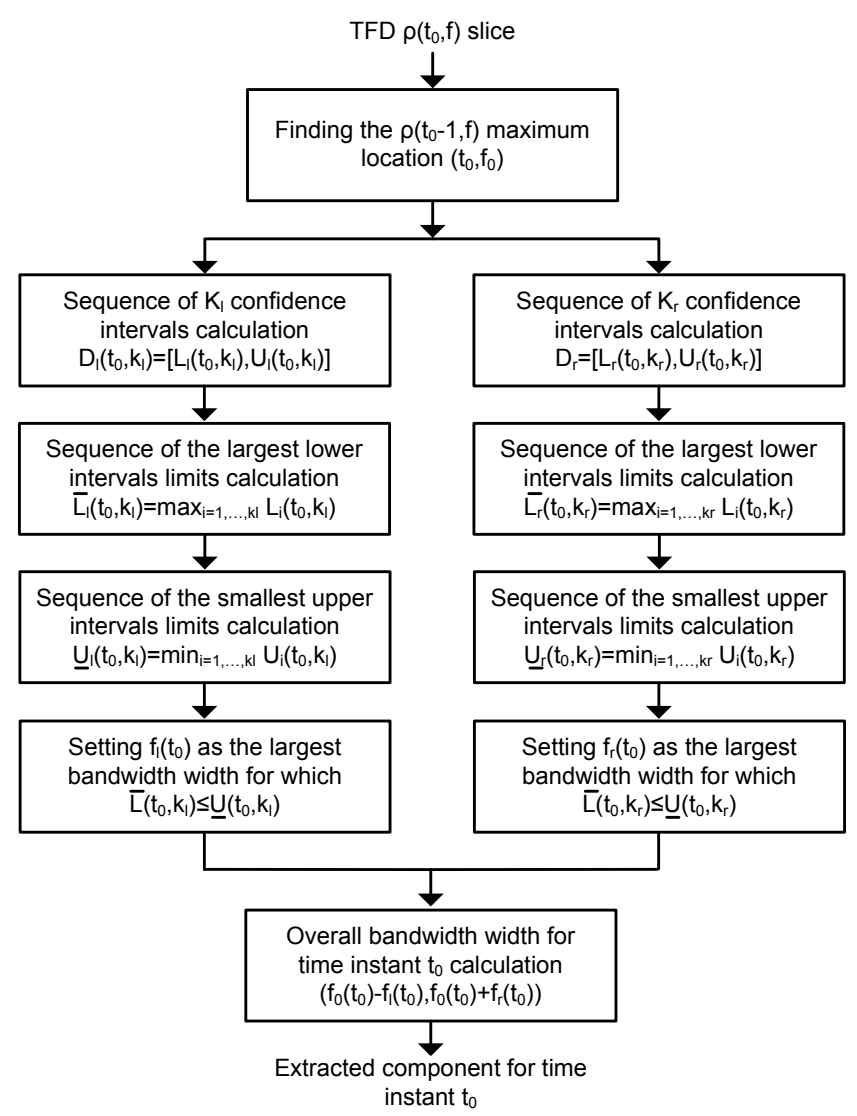

Fig. 2. Adaptive bandwidths selection flowchart.

giving the subbandwidth size $f_{l}^{+}\left(t_{0}\right)$ as the largest one for which it is still true that:

$$
\bar{L}_{l}\left(t_{0}, k_{l}\right) \leq \underline{U}_{l}\left(t_{0}, k_{l}\right)
$$

and the subbandwidth size $f_{r}^{+}\left(t_{0}\right)$ as the largest one for which it is true that:

$$
\bar{L}_{r}\left(t_{0}, k_{r}\right) \leq \underline{U}_{r}\left(t_{0}, k_{r}\right)
$$

The $f_{l}^{+}\left(t_{0}\right)$ and $f_{r}^{+}\left(t_{0}\right)$ were shown to be the subbandwidth lengths closest to the optimal lengths $f_{l}^{*}\left(t_{0}\right)$ and $f_{r}^{*}\left(t_{0}\right)$, respectively, giving the optimal bias to variance tradeoff and minimizing the estimation mean square error (MSE) [3].

\section{SIMULATION RESULTS AND DISCUSSION}

The proposed method for components localization and extraction was applied to a noisy three component signal (with the length $N=128)$ of the form $x(n)=z_{1}(n)+z_{2}(n)+$ $z_{3}(n)+\epsilon(n)$, where $z_{m}(n)=A_{m} \exp \left(j \phi_{m}(n)\right)\left(A_{m}=1\right)$ is a signal component, and $\epsilon(n)$ is complex-valued additive Gaussian noise. The signal contains two sinusoidal frequency modulated (FM) components and one linear FM component with different time supports (which partially overlap). The IF laws of the components are: $\omega_{1}(n)=0.35+0.05 \cos (2 \pi(n-$ $\left.\left.N_{1} / 2\right) / N_{1}-\pi / 2\right), \omega_{2}(n)=0.05+0.3(n-1) /\left(N_{2}-1\right)$, and $\omega_{3}(n)=0.075+0.025 \cos \left(2 \pi\left(n-N_{3} / 2\right) / N_{3}-\pi / 2\right)$ (the component lengths are $N_{1}=96, N_{2}=48$, and $N_{3}=48$ ).

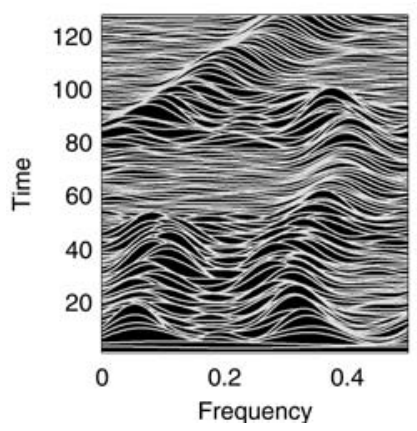

(a)

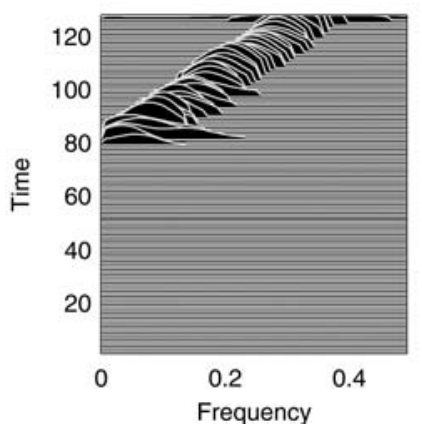

(c)

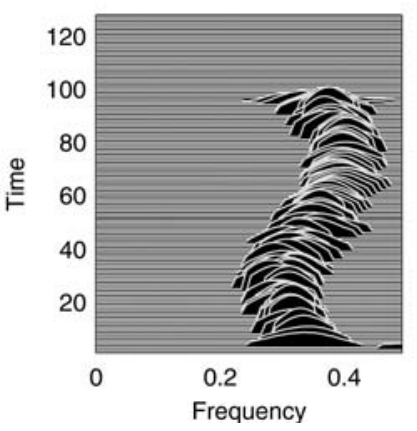

(b)

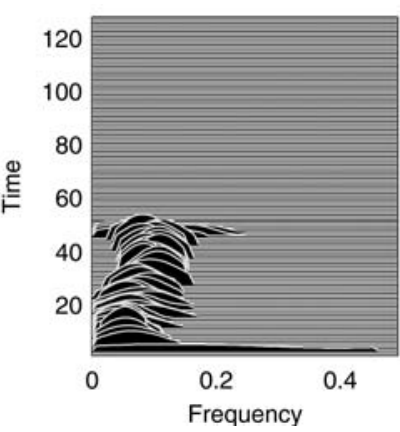

(d)
Fig. 3. (a) Noisy signal components extraction (MBD calculated using rectangular time and lag windows of length $h=9$, $\mathrm{SNR}=10, \Gamma=25, \epsilon_{c}=0.25, \epsilon_{d}=0.25, N=128, \beta=0.1$ as in [2]). (b) First component extracted. (c) Second component extracted. (d) Third component extracted.

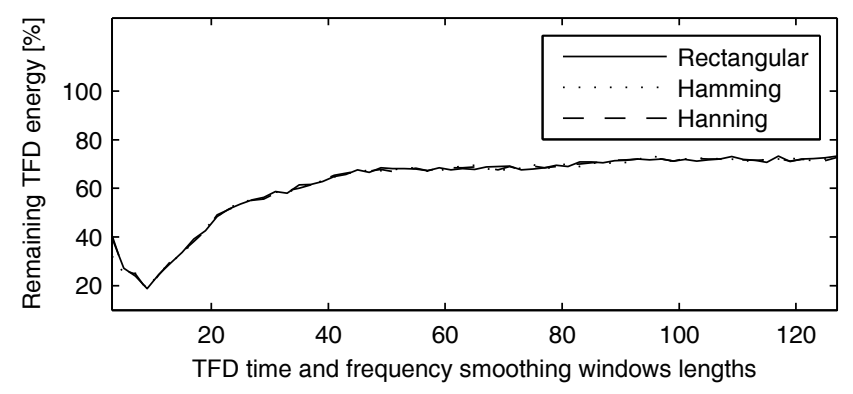

Fig. 4. Remaining MBD energy after components extraction as a function of the MBD time and lag window lengths ( $\Gamma=$ $25, \mathrm{SNR}=10, \epsilon_{c}=0.25, N=128, m=100, \beta=0.1$ as in [2]).

The method's component extraction efficiency has been analyzed for various time and lag window types and lengths $h$ used in the MBD calculation, as well as for different SNRs.

The results of the components extraction using the proposed adaptive method are given in Fig. 3, showing that each component was precisely localized and extracted. However, the method performance is effected by the TFD cross-terms suppression and TF resolution capabilities which depend on the MBD time and lag window lengths [2]. The remaining MBD energy after the components extraction (averaged over $m=$ 


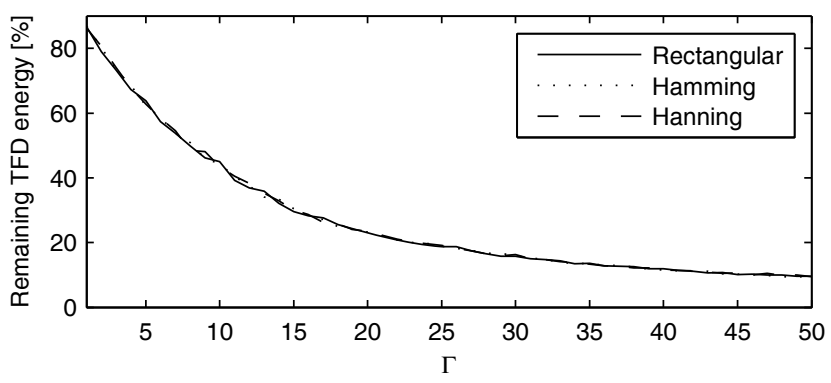

Fig. 5. Remaining MBD energy after components extraction as a function of $\Gamma$ (MBD calculated using the time and lag windows of length $h=9, \mathrm{SNR}=10, \epsilon_{c}=0.25, m=100$, $\beta=0.1$ as in [2]).

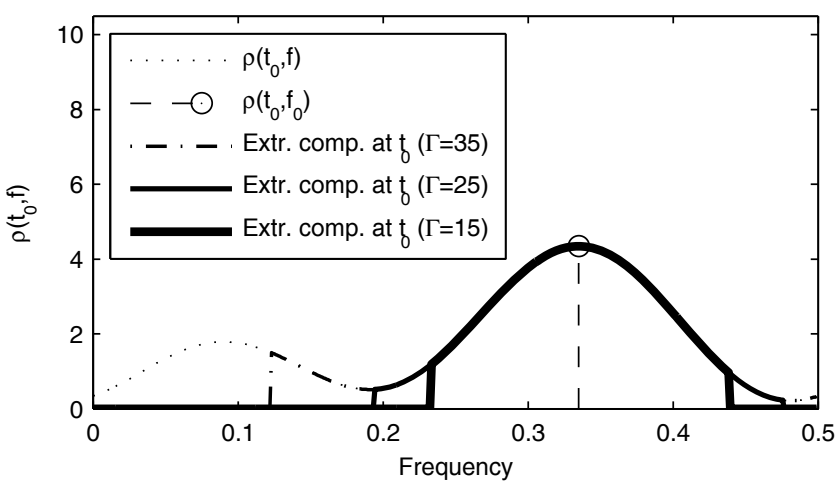

Fig. 6. Slice $\rho\left(t_{0}, f\right)$ and the component extraction for the time instant $t_{0}$ and different $\Gamma$ values.

Table 1. Remaining TFD energy after components extraction for different SNRs and $\Gamma$ values (MBD calculated using the rectangular time and lag windows of length $h=9, \epsilon_{c}=0.25$, $N=128, m=100, \beta=0.1$ as in [2]).

\begin{tabular}{|c|c|c|c|c|}
\hline$\Gamma$ & 2 & 5 & 10 & 15 \\
\hline 5 & 72.49 & 65.67 & 61.94 & 70.96 \\
\hline 10 & 56.37 & 44.79 & 42.73 & 54.57 \\
\hline 15 & 44.79 & 35.26 & 30.30 & 41.62 \\
\hline 20 & 38.86 & 26.46 & 23.23 & 32.52 \\
\hline 25 & 35.34 & 24.90 & 18.82 & 27.31 \\
\hline 50 & 25.54 & 22.34 & 9.70 & 13.80 \\
\hline
\end{tabular}

100 Monte Carlo simulation runs) as a function of the MBD time and lag window lengths and the ICI parameter $\Gamma$ for the rectangular, Hamming, and Hanning window types is shown in Figs. 4 and 5, respectively.

Table 1 gives the results of the remaining TFD energy after the components extraction for different SNRs and $\Gamma$ values. The choice of $\Gamma$ affects the time-varying bandwidth size selection, and thus the component extraction quality, as shown in Fig. 6. Too small $\Gamma$ values give undersized bandwidth lengths which result in omitting some parts of the considered component, hence increasing the remaining TFD energy after the components extraction. On the other hand, too large $\Gamma$ values result in oversized bandwidth lengths which may lead to inaccurate component selection (e.g. selecting segments of neighboring components), although the remaining MBD energy after the extraction would be smaller than the one obtained using a smaller $\Gamma$ value. Thus, when evaluating the method performance, beside the remaining TFD energy after the component extraction, one should also take into consideration whether the components are correctly localized, as ensured by this proposed algorithm.

\section{CONCLUSION}

This paper presents an automatic adaptive method to localize and extract signal components from a noisy multicomponent signal TFD. The method is based on the asymmetrical adaptive bandwidth selection for each time instant and each component apart. In order to get the proper bandwidth size, the ICI rule was used. The method's performance was analyzed for different SNRs and various time and lag window lengths and types used in the MBD calculation. The presented results show that the method is an efficient tool for automatic components extraction of noisy multicomponent signals in the TF domain.

\section{REFERENCES}

[1] B. Boashash, Ed., Time Frequency Signal Analysis and Processing: A Comprehensive Reference, Elsevier, Oxford, UK, 2003.

[2] Z. M. Hussain and B. Boashash, "Adaptive instantaneous frequency estimation of multicomponent FM signals using quadratic time-frequency distributions," Signal Processing, IEEE Transactions on, vol. 50, no. 8, pp. 18661876, Aug 2002.

[3] J. Lerga and V. Sucic, "Nonlinear IF estimation based on the pseudo WVD adapted using the improved sliding pairwise ICI rule," Signal Processing Letters, IEEE, vol. 16, no. 11, pp. 953-956, Nov 2009.

[4] B. Barkat and K. Abed-Meraim, "Algorithms for blind components separation and extraction from the timefrequency distribution of their mixture," EURASIP J. Appl. Signal Process., vol. 2004, pp. 2025-2033, 2004.

[5] J Lerga, V. Sucic, and B. Boashash, "An efficient algorithm for instantaneous frequency estimation of nonstationary multicomponent signals in low SNR," EURASIP J. Adv. Signal Process., vol. 2011, 2011.

[6] J. Lerga, M. Vrankic, and V. Sucic, "A signal denoising method based on the improved ICI rule," Signal Processing Letters, IEEE, vol. 15, pp. 601-604, 2008. 